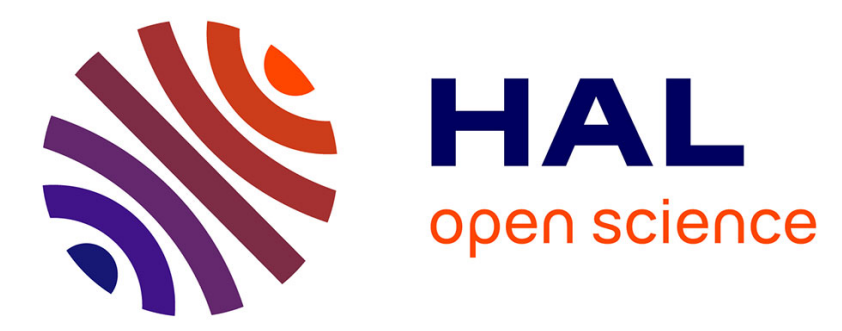

\title{
Does early verbal fluency decline after STN implantation predict long-term cognitive outcome after STN-DBS in Parkinson's disease?
}

Alaina Borden, David Wallon, Romain Lefaucheur, Stéphane Derrey, Damien Fetter, Marc Verin, David Maltête

\section{To cite this version:}

Alaina Borden, David Wallon, Romain Lefaucheur, Stéphane Derrey, Damien Fetter, et al.. Does early verbal fluency decline after STN implantation predict long-term cognitive outcome after STNDBS in Parkinson's disease?. Journal of the Neurological Sciences, 2014, 346 (1), pp.299-302. 10.1016/j.jns.2014.07.063 . hal-01075308

HAL Id: hal-01075308

https://hal-univ-rennes1.archives-ouvertes.fr/hal-01075308

Submitted on 17 Oct 2014

HAL is a multi-disciplinary open access archive for the deposit and dissemination of scientific research documents, whether they are published or not. The documents may come from teaching and research institutions in France or abroad, or from public or private research centers.
L'archive ouverte pluridisciplinaire HAL, est destinée au dépôt et à la diffusion de documents scientifiques de niveau recherche, publiés ou non, émanant des établissements d'enseignement et de recherche français ou étrangers, des laboratoires publics ou privés. 


\section{CLINICAL SHORT COMMUNICATION}

\section{Does early verbal fluency decline after STN implantation predict long-term cognitive outcome after STN-DBS in Parkinson's disease?}

Alaina Borden, MD,${ }^{1}$ David Wallon, MD,${ }^{1,3}$ Romain Lefaucheur, MD,${ }^{1}$ Stéphane Derrey, MD, $\mathrm{PhD},{ }^{2}$ Damien Fetter, MD, ${ }^{1}$ Marc Verin, MD $\mathrm{PhD},{ }^{4,5}$ David Maltête, MD, PhD. ${ }^{1,3}$

${ }^{1}$ Department of Neurology, Rouen University Hospital and University of Rouen, France

${ }^{2}$ Department of Neurosurgery, Rouen University Hospital and University of Rouen, France

${ }^{3}$ INSERM U1079, Rouen Faculty of Medicine, France

${ }^{4}$ Department of Neurology, University Hospital of Rennes

${ }^{5}$ EA 4712 "Behavior and Basal Ganglia," Rennes 1 University

Correspondence and reprint requests to:

Pr. David Maltête,

Department of Neurology,

Rouen University Hospital

76031 Rouen, Cedex, France

Tel.: + (33) 2328887 40; Fax: + (33) 232888741

E-Mail: david.maltete@ chu-rouen.fr

Word count: abstract 199 words; text 2689 words; 2 tables; 33 references.

Running title: STN implantation and cognitive microlesion effect

Disclosure: The authors confirm that there was no conflict of interest.

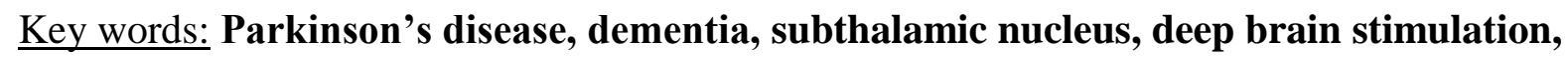
verbal fluency, microlesion. 


\section{AUTHORS' ROLES:}

Alaina Borden: acquisition of data, organization, writing of the draft.

David Wallon: acquisition of data, review and critique.

Romain Lefaucheur: acquisition of data, review and critique.

Stéphane Derrey: acquisition of data, review and critique.

Damien Fetter: acquisition of data, review and critique.

Marc Vérin: acquisition of data, review and critique.

David Maltête: conception and design, writing of part the draft, supervision.

\section{CONFLICTS OF INTEREST / DISCLOSURE OF ALL AUTHORS}

All authors and contributors agree to the conditions outlined in the Authorship and contributorship section of the Information for Authors. Include statement of responsibility for clinical trial data.

Alaina Borden reports no disclosures.

David Wallon reports no disclosures.

Romain Lefaucheur reports no disclosures.

Stéphane Derrey reports no disclosures.

Damien Fetter reports no disclosures.

Marc Verin reports no disclosures.

David Maltête reports no disclosures. 


\section{ABSTRACT}

Backgrounds: An early and transient verbal fluency (VF) decline and impairment in frontal executive function, suggesting a cognitive microlesion effect may influence the cognitive repercussions related to subthalamic nucleus deep brain stimulation (STN-DBS.)

Methods: Neuropsychological tests including semantic and phonemic verbal fluency were administered both before surgery (baseline), the third day after surgery (T3), at six months (T180), and at an endpoint multiple years after surgery (Tyears).

Results: Twenty-four patients (mean age, $63.5 \pm 9.5$ years; mean disease duration, $12 \pm 5.8$ years) were included. Both semantic and phonemic VF decreased significantly in the acute post-operative period $(44.4 \pm 28.2 \%$ and $34.3 \pm 33.4 \%$, respectively) and remained low at 6 months compared to pre-operative levels (decrease of $3.4 \pm 47.8 \%$ and $10.8 \pm 32.1 \%)(P<$ 0.05). Regression analysis showed phonemic VF to be an independent factor of decreased phonemic VF at six months. Age was the only independent predictive factor for incident Parkinson's disease dementia $(\mathrm{PDD})(\mathrm{F}(4,19)=3.4, P<0.03)$.

Conclusion: An acute post-operative decline in phonemic VF can be predictive of a longterm phonemic VF deficit. The severity of this cognitive lesion effect does not predict the development of dementia which appears to be disease-related. 


\section{Introduction}

Bilateral subthalamic nucleus deep brain stimulation (STN-DBS) in selected patients with advanced Parkinson's disease (PD) has been shown to improve motor symptoms and quality of life [1]. Even though the procedure is not considered to cause major cognitive sideeffects, it is associated with changes in executive function, in particular decreased verbal fluency (VF) [2]. Several factors are thought to predict cognitive decline following STNDBS, such as advanced age, axial signs, levodopa resistant symptoms, visual hallucinations, vascular lesion load, and poor baseline cognitive performance [3-4]. Higher formal education, higher levodopa equivalent dose, and younger age at onset also correlate with cognitive worsening after STN-DBS in PD [5].

A surgical motor microlesion effect appearing in the immediate post-operative period has been shown to be predictive of motor improvement 6 months after STN-DBS [6]. Similarly, an early and transient VF decline and impairment in frontal executive function has been described, suggesting a cognitive microlesion effect that may be partly responsible for the cognitive repercussions related to STN-DBS [7-9]. Whether this early cognitive impairment influences long-term cognitive performances and the incidence of Parkinson's disease dementia (PDD) after STN-DBS remains unknown.

In this study, we evaluated VF in 26 consecutive PD at baseline, in the immediate post-operative period prior to activating the pulse generator, 6 months after surgery, and at the end of the observation period (up to eight years after surgery.) We assessed whether the decline in VF present immediately after STN-DBS could be predictive of VF assessed 6 months after surgery and the apparition of PDD years after the surgery. 


\section{Materials and Methods}

\section{Patients}

Twenty-six consecutive patients (mean age, $57.9 \pm 8.5$ years; range, 37-79 years) with advanced levodopa-responsive PD (Hoehn and Yahr score $\geq 3$; mean disease duration, $11.4 \pm$ 3.5 years; range, 5-19 years) referred for STN stimulation were enrolled. The selection criteria were clinically diagnosed PD, severe levodopa-related complications despite optimal adjustment of antiparkinsonian medication, no surgical contraindications, and no dementia or major ongoing psychiatric illness.

\section{Surgical Procedure and Post-operative Management}

The surgical procedure has been previously described [10]. All recordings were performed on five parallel trajectories. The definitive quadripolar electrode (DBS type 3389, Medtronic, Minneapolis, Mn, USA) was positioned so that at least two contacts were located within the STN according to the best perioperative electrophysiological recordings and motor improvement and the fewest side effects. During the same session, the whole procedure was repeated to implant the contralateral STN. The day following the STN implantation, the programmable pulse generator (Kinetra $\Theta$, Medronic) was implanted under general anesthesia in the subclavicular region. All the patients gave their informed consent to their participation in the study and the protocol was approved by our local ethic committee.

\section{Neuropsychological testing}

Neuropsychological testing, i.e. the Mattis Dementia Rating Scale (MDRS), the Stroop test, free and cued recall, the Wisconsin card sorting test, digit and spatial spans, serial ordering, and MMS were administrated both before surgery (baseline), after surgery at six 
months (T180), and at an endpoint multiple years after surgery (Tyears). The final evaluation occurred in 2012 or 2013 if no earlier proof of PDD was available. Semantic (animal names) and phonemic (words beginning with "p") VF performances were evaluated both before surgery (baseline) and, after surgery the third day (T3), at six months (T180), and at an endpoint multiple years after surgery (Tyears).

The baseline cognitive evaluation was performed one month before the intervention, in the on-drug condition. At T3, the cognitive evaluation was performed in the off- condition, i.e. $12 \mathrm{~h}$ withdrawal of dopaminergic treatment and with the programmable pulse generator switched off (off-drug/ off-stimulation). The six-month (T180) post-operative cognitive and the at-distance evaluations were performed in the on-drug/on-stimulation condition.

In addition, dementia analyses were conducted using the criteria established by Emre et al.[11] and DSM-IV-TR [12] independently to identify participants who met criteria for PDD, i.e. a dementia syndrome with insidious onset and slow progression, developing within the context of established Parkinson's disease and diagnosed by history, clinical, and mental examination defined as: impairment in more than one cognitive domain; representing a decline from premorbid level; deficits severe enough to impair daily life (social, occupational, or personal care), independent of the impairment ascribable to motor or autonomic symptoms.

The patients were assessed with the Confusion Assessment Method (CAM) after surgery at T3, and T180; a score of CAM > 2/4 was considered as a confusional state [13].

\section{Motor Evaluation}

Clinical evaluations were performed one month before and six months after surgery. Motor performance and complications of levodopa therapy were assessed using the UPDRS parts III, and IV, respectively. The percentage of motor improvement was determined in 
comparison to the preoperative off-drug condition. All dopaminergic drugs, expressed in dopa-equivalent daily dose, were recorded before and after surgery.

\section{Statistical analysis}

The different scores were expressed as mean value \pm SD. Scores obtained before surgery (baseline on-state) were compared with scores at T3 and T180 after surgery using an analysis of variance for repeated measures (ANOVA). VF decline was defined as the percentage of reduction (total number of words) between baseline and T3.

Baseline MDRS score, age at the time of DBS, and percentage of VF decline (total number of words) were included in a multiple regression to determine: i) firstly, which were predictive of the VF assessed six months after surgery; ii) secondly, which were predictive of the occurrence of PDD years after surgery.

Statistical analysis was performed with the STATISTICA version 7.1, StatSoft1, France.

\section{Results}

Twenty-four patients (mean age, $63.5 \pm 9.5$ years; range, $36-79$ years ; mean disease duration, $12 \pm 5.8$ years; range, $5-30$ years) were included. One patient was excluded because the evaluation three days after surgery could not be performed. A second patient was excluded because he had a positive CAM scale proving a confusional state at T3. This patient converted to dementia at six months. Table 1 shows the demographic characteristics of the study sample.

At 6 months, the UPDRS part III scores in the off-drug/on-stimulation and on-drug/onstimulation conditions significantly improved from the baseline (off-state) value respectively by $65 \%$ (range, $23-100 \%$ ) and $82 \%$ (range, $52-100 \%)(\mathrm{P}<0.05)$. The severity of motor 
fluctuations and dyskinesia as assessed by the UPDRS IV was significantly reduced by $62 \%$ (range, $-300-100 \%$ ) and 59\% (range, $-300-100 \%$ ), respectively. Dopaminergic drugs, expressed in dopa-equivalents, were reduced by 56\% (range, $8.3-100 \%$ ), from $1093 \pm 342$ $\mathrm{mg} /$ day preoperatively to $487 \pm 308 \mathrm{mg} /$ day at 6 months. The mean intensities were $2.9 \pm 0.3$ and 3. $\pm 0.4 \mathrm{~V}$, pulse width $63.8 \pm 10.1$ and $67.5 \pm 13.3 \mu$ s (respectively on the right and left sides), and frequency $141 \pm 25 \mathrm{~Hz}$.

Pre- and post-surgical values of semantic and phonemic VF are shown in Table 2. Number of total words was significantly reduced in the immediate post-operative period at T3 for both fluency tasks. Semantic VF decreased by $44.4 \pm 28.2 \%$ and phonemic VF by $34.3 \pm$ 33.4\% $(P<0.05)$. Repeated post-operative neuropsychological testing demonstrated reliable improvement from T3 to T180 on verbal fluency tasks. Nevertheless, both semantic and phonemic VF at T180 remained significantly decreased in comparison to baseline scores, respectively by $3.4 \pm 47.8 \%$ and $10.8 \pm 32.1 \%(P<0.05)$.

Nine patients fulfilled criteria for probable PDD at the end up follow-up, two of these within the first year following STN-DBS. New cases of PDD appeared throughout the followup period which lasted from five years and three months to eight years. Once a patient was considered to have fulfilled the criteria for PDD, no later neuropsychological tests were administered. All of the patients categorized as PDD met criteria proposed by both Emre and the DSM-IV-TR.

First, multiple regression analysis showed that the reduction of phonemic VF at T3 was an accurate independent predictor of the reduction of phonemic VF at $\operatorname{T} 180(\mathrm{~F}(3,20)=$ 3.92, $\mathrm{P}<0.01$ ). Secondly, multiple regression analysis showed that age was an independent predictive factor for incident PDD $(\mathrm{F}(4,19)=3.42, \mathrm{P}<0.03)$. Other variables did not appear as independent predictors. 


\section{Discussion}

In this study, we demonstrated that acute post-operative decline in phonemic VF can be predictive of a long-term phonemic VF deficit. However, the severity of this cognitive lesion is not correlated with the development of PDD.

Meta-analyses have revealed that patients who underwent STN-DBS demonstrated mild but significant cognitive changes in verbal memory and executive functions and moderate decline in both semantic and phonemic VF [14-15]. Changes in phonemic and semantic VF are recognized as the most common cognitive decline following surgery [16-17]. Nevertheless, the mechanisms leading to cognitive changes following STN-DBS remain obscure. Since the intermediate and ventral parts of the STN are involved in both cognitive and limbic processes, it has been postulated that diffusion of the electrical current may affect cognitive performance, including VF [18]. Accordingly, at ventral contacts, more tissue activation inside the STN has been associated with decreased phonemic fluency performances [19]. Conversely, evidence has been accumulating suggesting that post-operative cognitive impairment could be more related to surgical procedure and its anatomical consequences than to the stimulation $[8,20-21]$.

Previously, we reported an early and transient VF decline in eighteen PD patients just after STN implantation with the programmable pulse generator switched off (off-drug/ offstimulation) [8]. Since VF improves within the first 6 months following the surgical procedure, we hypothesized that the early VF impairment could above all reflect a cognitive microlesion effect. These findings corroborate a recent study that compared stimulation with implantation alone using the Saint Jude Medical constant-current device [20]. In this study, Okun et al. demonstrated that VF declines are related to the surgical procedure rather than the stimulation in STN-DBS. Another recent publication illustrated a greater decline in alternating VF performance in PD patients with STN-DBS as compared to patients that had 
not been operated [21]. Taken together, these results are consistent with reports which have found that both intraoperative electrophysiological recordings and multiple trajectories of the definitive quadripolar electrode may contribute to deterioration in global cognition and memory function in PD patients after STN implantation [22-23]. Interestingly, recent studies have shown an increased risk of global cognitive decline and working memory performance after STN-DBS in PD when the chronic stimulation lead passes through the head of the caudate [22].

In the present study, we found that the decline in phonemic VF was predictive of a continued decline in phonemic VF at six months. It is commonly believed that the temporal cortex supports word retrieval constrained by semantics while frontal regions support word retrieval constrained by phonology [24]. Thus, the microlesion effect along the trajectory of the electrode (passing through the dorsolateral prefrontal cortex) may be more pronounced on the phonemic VF task that relies solely on the frontostriatal network. As expected, the longterm outcome of phonemic VF following surgical procedure correlated with the early decline synonymous of microlesion.

Patients with PD have an increased risk of dementia compared to the general population. Thus, the likelihood of a patient with PD to develop dementia has been estimated at as high as forty percent with a cumulative prevalence of at least $75 \%$ ten years after diagnosis [25]. Cognitive impairment in PD is generally thought to affect predominantly executive and visuospatial functions but can touch various cognitive domains and has been found in patients at all stages of the disease. Patients with PDD present a dysexecutive profile of cognitive impairment where the most affected functions are attention, executive functions, and visuospatial functions, but memory can also be impacted [11].

Even though STN-DBS is generally considered to be a cognitively safe procedure in well-selected patients, several studies show an accelerated cognitive decline in the months 
following surgery, suggesting that a sub-group of patients may have a predisposition towards PDD following STN-DBS [26] However, few studies have tried to delineate predictive factors of PDD following STN-DBS. Notably, it has been reported that hippocampal atrophy is a predictor of dementia in PD patients converting to dementia after STN-DBS [26] Moreover, patients with advanced age, impaired baseline attention, and poorer levodopa response seem at greater risk for post-surgical cognitive decline [27, 28 ]. Since VF tests have been reported to be predictive of dementia in a community-based sample of non-operated PD patients, [29] we hypothesized that the long-term cognitive outcome following STN-DBS could be influenced by the severity of the early VF decline observed just after STN implantation. In our study, almost $35 \%(n=9)$ of patients fulfilled criteria for probable PDD at the end up follow-up, $22 \%(\mathrm{n}=2)$ of these within the first year following STN-DBS. This incidence rate of dementia following STN-DBS is high when compared to previous studies with a similar follow-up period [26-27]. However, this result is consistent with the incidence of dementia observed in the natural course of PD, suggesting that PDD in our patients implanted with STN-DBS is most likely disease-related [25]. The fact that we were not able to clearly show that an early decline in VF (synonymous of microlesion) can predict which PD patients will develop dementia also corroborates this hypothesis.

In accordance with previous findings, older patients were more likely to develop dementia [25]. An interesting observation is that two patients who had performed well on cognitive tests, but had experienced hallucinations before the procedure, rapidly developed dementia relative to the rest of the population. Cognitive dysfunction has been noted after all types of surgery in older patients, and early post-operative cognitive decline was identified as an independent risk factor for persistent cognitive decline in one study [30]. However, there is little evidence that post-operative cognitive dysfunction leads to dementia or that surgery and/or anesthesia accelerate cognitive decline [31]. 
Three limitations of this study need to be pointed out: First, the population is small in size and relatively heterogeneous, in particular with regard to age. In addition, one patient was excluded for confusion at T3 and another patient was not tested at that same time point, further limiting the population size. Second, we considered the reduction of the number of words during VF analysis in the early post-operative as the optimal value to reflect the cognitive microlesion effect. However, we compared VF in two different conditions before and after surgery, i.e. with or without dopaminergic treatment. Levodopa treatment has often been shown to improve executive function in PD [32]. Therefore, as the fluency tasks necessitate a high degree of cognitive flexibility, we cannot exclude the involvement of abrupt withdrawal of levodopa treatment in the early post-operative VF decline. Accordingly, we previously described that post-operative VF impairment at T3 improves 10 days after surgery with resumed dopamine-replacement therapy [8]. Nevertheless, the a significant persistent impairment in verbal fluency in the on-drug condition compared to the baseline on-state pleads in favor of a microlesion effect. These results are in keeping with previous studies that failed to correlate VF decline and levodopa equivalent daily dose decrease after STN-DBS [89, 15]. Moreover, other tests to explore executive dysfunction may have been considered as more pertinent but VF decline is recognized as the most common cognitive decline following STN-DBS surgery. Third, the length of follow-up was not entirely uniform. We consider however that over five years of follow-up is sufficient as any cases of dementia related to a stimulation-lead injury would have occurred early. Cases of dementia that occur multiple years after STN-DBS are most likely not related to the procedure and would be in any case indistinguishable from cases of dementia that are often part of the natural course of the disease. 


\section{Conclusion}

Our findings provide further information regarding the impact of the surgical procedure for STN-DBS on cognitive function over time. We demonstrated that the early decline in VF in the acute postoperative period can predict long-term VF deficits. Recently, STN-DBS was shown to improve quality of life in patients with early motor complications [33]. As teams are encouraged to implement DBS earlier in the course of the disease, more patients will be eligible for DBS before they are considered too old for the procedure. Therefore, the cognitive lesion effect merits to be studied in younger patients in order to better understand which patients are more likely to exhibit VF deficits. 


\section{Abbreviations.}

\section{VF: Verbal fluency}

STN- DBS: Subthalamic nucleus deep brain stimulation

PD: Parkinson disease

VAS: Visual Analogic Scale

UPDRS: Unified Parkinson Disease Rating Scale

BMI: Body Mass Index 


\section{REFERENCES}

[1] Deuschl G, Schade-Brittinger C, Krack P et al (2006) A randomized trial of deep-brain stimulation for Parkinson's disease. N Engl J Med 355:896-908.

[2] Sáez-Zea C, Escamilla-Sevilla F, Katati MJ, Mínguez-Castellanos A (2012) Cognitive Effects of Subthalamic Nucleus Stimulation in Parkinson's Disease: A Controlled Study. Eur Neurol 68:361-366.

[3] Daniels C, Krack P, Volkmann J et al (2010) Risk factors for executive dysfunction after subthalamic nucleus stimulation in Parkinson's disease. Mov Disord 25:1583-1589.

[4] Massano J, Garrett C (2012) Deep Brain Stimulation and Cognitive Decline in Parkinson's Disease: A Clinical Review. Front. Neurol 3:66.

[5] Heo JH, Lee KM, Paek SH, Kim MJ, Lee JY, Kim JY, Cho SI, Lim YH, Kim MR, Jeong SY, Jeon BS (2008) The effects of bilateral Subthalamic Nucleus Deep Brain Stimulation (STN DBS) on cognition in Parkinson disease. J Neurol Sci 273:19-24.

[6] Maltête D, Derrey S, Chastan N, Debono B, Gérardin E, Fréger P, Mihout B, Menard JF, Hannequin D (2008) Microsubthalamotomy: An immediate predictor of long-term subthalamic stimulation efficacy in Parkinson disease. Mov Disord 23:1047-1050. 
[7] Zangaglia R, Pachetti C, Pasotti C, Mancini F, Servello D, Sinforiani E, Cristina S, Sassi M, Nappi G (2009) Deep brain stimulation and cognitive functions in Parkinson's disease: A three-year controlled study. Mov Disord 24:1621-1628.

[8] Lefaucheur R, Derrey S, Martinaud O, Wallon D, Chastan N, Gérardin E, Hannequin D, Maltete D (2012) Early verbal fluency decline after STN implantation: Is it a cognitive microlesion effect? J Neurol Sci 321:96-99.

[9] Yamanaka T, Ishii F, Umemura A, Miyata M, Horiba M, Oka Y, Yamada K, Okita K, Matsukawa N, Ojika K (2012) Temporary deterioration of executive function after subthalamic deep brain stimulation in Parkinson's disease. Clin Neurol Neurosurg 114:347351.

[10] Derrey S, Maltete D, Chastan N, Debono B, Proust F, Gerardin E, Weber J, Mihout B, Fréger P (2008) Deep brain stimulation of the subthalamic nucleus in Parkinson's disease: usefulness of intraoperative radiological guidance. The Stereoplan Stereotact Funct Neurosurg $86: 351-358$.

[11] Emre M, Aarsland D, Brown R, et al (2007) Clinical diagnostic criteria for dementia associated with Parkinson's disease. Mov Disord 22:1689-1707.

[12] American Psychiatric Association. Diagnostic and Statistical Manuel of Mental Disorders. 4th ed. -- text revision (DSM IV-TR). Washington DC: American Psychiatric Association; 2000. 
[13] Inouye SK, van Dyck CH, Alessi CA, Balkin S, Siegal AP, Horwitz RI (1990) Clarifying Confusion: The Confusion Assessment Method: A New Method for Detection of Delirium. Ann Intern Med 113:941-948.

[14] Parsons TD, Rogers SA, Braaten AJ, Woods SP, Tröster AI (2006) Cognitive sequelae of subthalamic nucleus deep brain stimulation in Parkinson's disease: a meta-analysis. Lancet Neurol 5:578-588.

[15] Temel Y, Kessels A, Tan S, Topdag A, Boon P, Visser-Vanderwalle V (2006)

Behavioural changes after bilateral subthalamic stimulation in advanced Parkinson disease: A systematic review. Parkinsonism Relat Disord 12:265-272.

[16] De Gaspari, D, Siri C, Di Gioia, Antonini A, Isella V, Pizzolato A, Landi A, Vergani F, Gaini SM, Appollonio IM, Pezzoli G (2006) Clinical correlates and cognitive underpinnings of verbal fluency impairment after chronic subthalamic stimulation in Parkinson's disease. Parkinsonism Relat Disord 12:289-295.

[17] Witt K, Daniels C, Reiff J et al (2008) Neuropsychological and psychiatric changes after deep brain stimulation for Parkinson's disease: a randomised, multicentre study. Lancet Neurol 7:605-614.

[18] Schroeder U, Kuehler A, Lange KW, Haslinger B, Tronnier VM, Krause M, Pfister R, Boecker H, Ceballos-Baumann AO (2003) Subthalamic nucleus stimulation affects a frontotemporal network: a PET study. Ann Neurol 54:445-450. 
[19] Mikos A, Bowers D, Noecker AM, McIntyre CC, Won M, Chaturvedi A, Foote KD, Okun MS (2011) Patient-specific analysis of the relationship between the volume of tissue activated during DBS and verbal fluency. NeuroImage 54:S238-S246.

[20] Okun MS, Gallo BV, Mandybur G, et al (2012) Subthalamic deep brain stimulation with a constant-current device in Parkinson's disease: an open-label randomised controlled trial. Lancet Neurol 11:140-149.

[21] Marshall DF, Strutt AM, Williams AE, Simpson RK, Jankovic J, York MK (2012) Alternating verbal fluency performance following bilateral subthalamic nucleus deep brain stimulation for Parkinson's disease. Eur J Neurol 19:1525-1531.

[22] Witt K, Granert O, Daniels C, Volkmann J, Falk D, van Eimeren T, Deuschl G (2013) Relation of lead trajectory and electrode position to neuropsychological outcomes of subthalamic neurostimulation in Parkinson's disease: results from a randomized trial. Brain $136: 2109-2119$.

[23] Temel Y, Wilbrink P, Duits A, Boon P, Tromp S, Ackermans L, van KranenMastenbroek, Weber W, Visser-Vandewalle V (2007) Single electrode and multiple electrode guided electrical stimulation of the subthalamic nucleus in advanced Parkinson's disease. Neurosurgery 61:346-355. 
[24] Baldo JV, Schwartz S, Wilkins D, Dronkers NF (2006) Role of frontal versus temporal cortex in verbal fluency as revealed by voxel-based lesion symptom mapping. J Int Neuropsychol Soc 12:896-900.

[25] Aarsland D, Kurz MW (2010) The epidemiology of dementia associated with Parkinson disease. J Neurol Sci 289:18-22.

[26] Aybek S, Gronchi-Perrin A, Berney A, Chiuvé SC, Villemure JG, Burkhard PR, Vingerhoets FJ (2007) Long-term cognitive profile and incidence of dementia after STN-DBS in Parkinson's disease. Mov Disord 22:974-981.

[27] Smeding HMM, Speelman JD, Huizenga HM, Schuurman PR, Schmand B (2009) Predictors of cognitive and psychosocial outcome after STN DBS in Parkinson's Disease. J Neurol Neurosurg Psychiatry 82:754-760.

[28] Shalash A, Alexoudi A, Knudsen K, Volkmann J, Mehdorn M, Deuschl G (2014). The impact of age and disease duration on the long term outcome of neurostimulation of the subthalamic nucleus. Parkinsonism Relat Disord:20:47-52.

[29] Levy G, Jacobs DM, Tang MX, Côté LJ, Louis ED, Alfaro B, Mejia H, Stern Y, Mardek K (2002) Memory and executive function impairment predict dementia in Parkinson's disease. Mov Disord 17:1221-1226. 
[30] Abildstrom H, Rasmussen LS, Rentowl P, Hanning CD, Rasmussen H, Kristensen PA, Moller JT (2000) Cognitive dysfunction 1-2 years after non-cardiac surgery in the elderly. Acta Anaesthesiol Scand 44:1246-1251.

[31] Silverstein JH, Deiner SG (2013) Perioperative delirium and its relationship to dementia. Prog Neuropsychopharmacol Biol Psychiatry 43:108-115.

[32] Kulisevsky J, Garcia-Sanchez C, Berthier ML, Barbanoj M, Pascual-Sedano B, Gironell A, et al. Chronic effects of dopaminergic replacement on cognitive function in Parkinson's disease: a two-year follow-up study of previously untreated patients. Mov Disord. 2000;15:613-26.

[33] Schuepbach WM, Rau J, Knudsen K et al (2013) Neurostimulation for Parkinson's Disease with Early Motor Complications. N Engl J Med 368:610-622. 


\section{Legends}

TABLE 1. Demographic characteristics at baseline

Values are given as mean \pm standard deviation.

Dopaminergic drugs are expressed in dopa-equivalent daily dose.

Table 1.

Demographic characteristics at baseline.

\begin{tabular}{|l|l|}
\hline Age, years & PD $(n=24)$ \\
\hline Men/women & $63.5 \pm 9.5$ \\
\hline Disease duration, years & $15 / 9$ \\
\hline Dopaminergic medication (mg/day) & $1093 \pm 342$ \\
\hline Hoehn and Yahr "off" staging & $3 \pm 0.6$ \\
\hline MDRS score & $12 \pm 5.8$ \\
\hline
\end{tabular}

Values are given as mean \pm standard deviation.

Dopaminergic drugs are expressed in dopa-equivalent daily dose.

TABLE 2. Pre and post-surgical verbal fluency

Values are given as mean \pm standard deviation.

Comparison of scores obtained before surgery (baseline on-state) and respectively at T3 and T180 after surgery used an analysis of variance for repeated measures (ANOVA). 
${ }^{*} P<0.05$ compared to baseline.

Table 2.

Pre and post-surgical verbal fluency.

\section{Preoperative Postoperative}

Baseline T3 T180

Semantic fivency

\begin{tabular}{l|l|l|l|}
\hline Total words & $24.3 \pm 7.4$ & $13 \pm 7^{*}$ & $21.5 \pm 6.9^{*}$ \\
\hline \% reduction & & $44.4 \pm 28.2$ & $3.4 \pm 47.8$
\end{tabular}

Phonemic fuency

\begin{tabular}{l|l|l|l} 
Total words & $18.3 \pm 6.7$ & $11.7 \pm 6^{*}$ & $15.5 \pm 5.8^{*}$
\end{tabular}

\begin{tabular}{l|l|l} 
\% reduction & $34.3 \pm 33.4$ & $10.8 \pm 32.1$
\end{tabular}

Values are given as mean \pm standard deviation.

Comparison of scores obtained before surgery (baseline on-state) and respectively at T3 and T180 after surgery used at analysis of variance for repeated measures (ANOVA).

* $\quad P<0.05$ compared to baseline. 